Research Paper

\title{
The impacts of single nucleotide polymorphisms in genes of cell cycle and NF-KB pathways on the efficacy and acute toxicities of radiotherapy in patients with nasopharyngeal carcinoma
}

\author{
Chengxian Guo ${ }^{1, *}$, Yuling Huang ${ }^{2, *}$, Jingjing $\mathbf{Y u}^{1, *}$, Lijuan Liu ${ }^{3}, X_{i a o c h a n g ~ G o n g}{ }^{2}$, \\ Min Huang ${ }^{2}$, Chunling Jiang ${ }^{2}$, Yulu Liao ${ }^{2}$, Lihua Huang ${ }^{4}$, Guoping Yang ${ }^{1}$, Jingao Li $^{2}$ \\ ${ }^{1}$ Center of Clinical Pharmacology, The Third Xiangya Hospital, Central South University, Changsha, Hunan 410013, China \\ ${ }^{2}$ Department of Radiation Oncology, Jiangxi Cancer Hospital, Nanchang, Jiangxi 330029, China \\ ${ }^{3}$ Department of Pharmacy, Jiangxi Cancer Hospital, Nanchang, Jiangxi 330029, China \\ ${ }^{4}$ Center for Medical Experiments, The Third Xiangya Hospital, Central South University, Changsha, Hunan 410013, China \\ *These authors have contributed equally to this work \\ Correspondence to: Jingao Li, email: lijingao@hotmail.com \\ Keywords: nasopharyngeal carcinoma (NPC), cell cycle, NF-KB pathway, single nucleotide polymorphism (SNP), clinical responses \\ Received:December 05, $2016 \quad$ Accepted: February 06, $2017 \quad$ Published: March 02, 2017 \\ Copyright: Guo et al. This is an open-access article distributed under the terms of the Creative Commons Attribution License \\ (CC-BY), which permits unrestricted use, distribution, and reproduction in any medium, provided the original author and source \\ are credited
}

\section{ABSTRACT}

Radiotherapy is one of the primary choices for the treatment of nasopharyngeal carcinoma (NPC) and may result in severe radiotoxicities on normal tissues. Single nucleotide polymorphisms (SNPs) in genes of cell cycle and NF-кB pathways have been linked with the prognoses of various cancers. The aim of this study was to explore whether SNPs of genes involved in cell cycle and NF-кB pathways are associated with responses to radiotherapy in NPC patients. We selected 3 SNPs in cell cycle pathway and 5 SNPs in NF-кB pathway and genotyped them in 154 NPC patients treated with radiotherapy. Multivariate logistic regression was used to determine the association of these 8 SNPs with the responses to radiotherapy. We observed that cyclin-dependent kinase inhibitor gene CDKN2A rs3088440 was significantly related with a poorer treatment efficacy on the primary tumor and cervical lymph node after radiotherapy, and also with a decreased risk of grade 3-4 acute radiation-induced myelosuppression. In some subgroups, cyclin D1 gene CCND1 rs9344 and inhibitor of KB kinase gene IKBKB rs12676482 were related with the grade 3-4 acute radiationinduced myelosuppression, and CCND1 rs9344 was also associated with grade 3-4 acute radiation-induced oral mucositis. The current results reveal that SNPs in genes of cell cycle pathwayand NF-кB pathway have the potential to predict the clinical responses to radiotherapy for NPC patients.

\section{INTRODUCTION}

Nasopharyngeal carcinoma (NPC) is one of the common head and neck cancers originating from the epithelial lining of the nasopharynx [1]. With a notably ethnically and geographically distributed imbalance, the incidence of NPC is much higher in southern China than Western countries [2]. Radiotherapy is one of the primary choices for the treatment of NPC due to the deep-rooted histological location and relative radiosensitivity of the carcinoma [3]. Despite the development of advanced radiotherapy techniques such as intensity-modulated radiotherapy (IMRT), a certain number of patients may experience radioresistance, which causes the distant metastasis or recurrence of the tumor. Since radiation may effect on normal tissues, patients treated with radiotherapy often suffer some complications like dermatitis, oral mucositis, dysphagia and hoarseness, which severely reduce 
the patients' life quality and even reduce the treatment efficacy due to frequent dose adjustment and delays of radiotherapy $[4,5]$. Moreover, the clinical outcome and the incidence of complications are heterogeneous among patients, which can be attributed to not only demographic and environmental factors, but also genetic factors [6].

With the rapid development of radiogenomics, a number of studies have illuminated the significant association between genetic variants with the efficacy and toxicity of radiotherapy in patients with NPC [7-12]. For example, Cheuk's preliminary results indicated that XRCC3 rs861539 polymorphism may be associated with increased risk of radiation-induced fibrosis in NPC patients [13]. Moreover, Li et al. have found that XRCC1 polymorphism affecting $399 \mathrm{Gln}$ is related with radiation-induced dermatitis and mucositis in NPC patients, and carriers of XRCC1 399Gln/Arg coding genotype had a higher risk of severe acute dermatitis and oral mucositis [14].

Cell cycle is the most essential physiological process, and dysregulation of normal cell cycle control has been implicated in the pathogenesis of most human cancers [15]. Main proteins involved in cell cycle regulation are cyclins, cyclin-dependent kinases (CDKs) and cyclin-dependent kinase inhibitors (CDKNs) [16]. Cyclin D1 encoded by the $C C N D 1$ gene is a key positive regulatory protein of the G1/S phase in the cell cycle. Variations of CCND1 have been detected in many cancers and CCND1 rs9344 polymorphism has been frequently reported to be related with several cancers including NPC [17-20]. One study found that the $\mathrm{G}$ allele of CCND1 rs9344 polymorphism was associated with a decreased risk of developing NPC [21]. The cyclindependent kinase inhibitor $\mathrm{p} 21$ encoded by $C D K N 1 A$ gene inhibits the phosphorylation of Retinoblastoma protein (Rb) by binding to cyclin-cdk complexes: cyclinE-cdk2, cyclinA-cdk2, and cyclinD-cdk4 [22]. CDKN1A rs1059234 polymorphism was found to be associated with increased risk of head and neck squamous cell carcinoma (HNSCC) [23]. Another tumor suppressor gene, $C D K N 2 A$, encoding two proteins $\mathrm{p} 14 \mathrm{ARF}$ and $\mathrm{p} 16 \mathrm{INK} 4 \mathrm{a}$, has also been reported to be associated with the development of several cancers, such as HNSCC [24, 25], cervical neoplasia [26] and glioma [27].

In addition, nuclear factor-kappa $\mathrm{B}(\mathrm{NF}-\kappa \mathrm{B})$ has also been involved with cancer biology through regulation of proliferation factors such as CCND1 [28]. Some SNPs of genes involved in NF- $\kappa \mathrm{B}$ signaling pathway have been frequently found associated with several cancers [29, 30]. For example, Hein et al. reported that individuals with two minor alleles of SNP rs1054016 of RANK gene (coding the receptor activator of $\mathrm{NF}-\kappa \mathrm{B}$ ) had a more favorable prognosis than those with at least one common allele in patients with breast cancer [31]. Recently, one study found that multiple loss-of-function mutations were identified in several NF- $\kappa \mathrm{B}$ signaling negative regulators tumor necrosis factor a-induced protein 3 (TNFAIP3), NF-кB inhibitor alpha (NFKBIA), and cylindromatosis (CYLD) in NPC. And the mutations in NFKBIA had a noticeable impact on NPC cell growth, which revealed the association between $\mathrm{NF}-\kappa \mathrm{B}$ signaling pathway and NPC [32]. Canonical NF- $\mathrm{NB}$ signaling is performed by a series of positive regulators such as tumor necrosis factor receptor-associated factor-6 (TRAF6) and negative regulators such as TNFAIP3 interacting protein 1 (TNIP1). Inhibitor of $\kappa \mathrm{B}$ kinase (IKK) $\beta$ encoded by $I K B K B$ gene is a kinase subunit of the IKK complex and plays a vital role in the activation of canonical NF- $\kappa B$ signaling pathway [33]. $N F-\kappa B I L 1$ gene encodes the NF$\kappa \mathrm{B}$ inhibitor-like protein 1 that represents a novel member of the inhibitor of $\kappa \mathrm{B}$ proteins (I $\mathrm{BBs}$ ) family, which prevent the nuclear translocation of NF- $\kappa B$ [34]. TNIP1 inhibits NF- $\kappa \mathrm{B}$ signaling pathway by cooperating with TNFAIP3 [35]. Some SNPs of aforementioned genes involved in NF$\kappa \mathrm{B}$ signaling pathway have been identified and linked with several diseases [36-39].

However, there has been no study that explores whether genetic polymorphisms of aforementioned cell cycle regulation and $\mathrm{NF}-\kappa \mathrm{B}$ signaling related genes are associated with the sensibility to radiotherapy in NPC patients. We hypothesized that some potentially functional SNPs of genes in cell cycle pathway and NF- $\kappa$ B pathway might have prognostic values for NPC patients treated with radiotherapy. According to previously published literatures, we chose 3 SNPs (CCND1 rs9344, CDKN1A rs1059234 and $C D K N 2 A$ rs3088440) in cell cycle pathway and 5 SNPs (IKBKB rs12676482, TRAF6 rs4755453 and rs5030437, TNIP1 rs10036748 and $I \kappa B L$ rs2071592) in NF- $\kappa \mathrm{B}$ pathway. The aim of this present study is to evaluate the association of the 8 SNPs with the efficacy and acute toxic reactions after radiotherapy in NPC patients and to find some novel genetic markers for the prognosis of NPC patients treated with radiotherapy.

\section{RESULTS}

\section{Clinical characteristics, radiotherapy responses and genotyping of the patients}

This study population consisted of 106 males and 48 males, with a mean age of 51 (ranging from 14 to 81 ). Thirty-two $(20.8 \%)$ patients were treated with radiotherapy alone, and 122 patients (79.2\%) were also treated with chemoradiotherapy. The general demographics and clinic pathologic characteristics as well as the clinical outcomes after radiotherapy of the 154 patients with NPC are summarized in Table 1. Because of some data missing, there were only data of 125 patients for curative efficacy at the cervical lymph node after radiotherapy. Overall, there were 29 (18.8\%) and 17 (13.6\%) patients who did not get $\mathrm{CR}$ after radiotherapy at their primary tumors and cervical lymph nodes, respectively. In the case of toxic reactions, 6 (3.9\%), $71(46.1 \%)$ and $32(20.8 \%)$ patients experienced grade 3-4 acute radiation-induced dermatitis, oral mucositis, and myelosuppression, respectively. The associations of all clinical factors with the responses to radiotherapy are shown 
Table 1: The characteristics and clinical outcomes of the patients

\begin{tabular}{lc}
\hline Characteristics & Patients (\%) (n=154) \\
\hline Gender & $106(68.8)$ \\
male & $48(31.2)$ \\
female & \\
Age & $14-81$ \\
range & $51 \pm 12$ \\
mean \pm SD & \\
BMI & $16.90-32.00$ \\
range & $22.79 \pm 3.27$ \\
mean \pm SD & \\
Smoking & $70(45.5)$ \\
yes & $84(54.5)$ \\
no & \\
Drinking & $38(24.7 \%)$ \\
yes & $116(75.3 \%)$ \\
no & \\
Chemotherapy & $32(20.8 \%)$ \\
no & $122(79.2 \%)$ \\
Non-CR after radiotherapy & \\
primary tumor & $29(18.8 \%)$ \\
lymph node & $17(13.6 \%)$ \\
Grade 3-4 radiation-induced toxic reactions & \\
dermatitis & $6(3.9 \%)$ \\
myelosuppression & $71(46.1 \%)$ \\
\hline
\end{tabular}

Abbreviations: BMI, body mass index; CR, complete remission.

in Supplementary Tables 1 and 2. Patients getting CR at the cervical lymph node were significantly older than those not getting $\mathrm{CR}(P=0.046)$. And compared with radiotherapy alone, patients treated with chemoradiotherapy had a higher risk of grade 3-4 myelosuppresion $(P=0.006)$. The results of genotyping and the allele type of each SNP in the 154 patients are shown in Table 2. All minor allele frequencies (MAFs) of 8 SNPs were $>0.10$ and all genotype distributions were in accordance with Hardy-Weinberg equilibrium.

\section{Associations between 8 SNPs and the efficacy of radiotherapy}

The associations between selected 8 SNPs and the efficacy at the primary tumor and cervical lymph node after radiotherapy are shown in Tables 3 and 4, respectively. $C D K N 2 A$ rs3088440 polymorphism was significantly associated with poorer efficacy at the primary tumor and cervical lymph node after radiotherapy in multiplicative, additive and dominant models after adjusted by age, gender, BMI, smoking and drinking status, TNM stage and with/ without chemotherapy. In detail, compared with the $G$ allele, patients with the minor A allele of this SNP had a 2-fold and 3-fold higher risk to get non-CR at the primary tumor (OR: 2.20, P: 0.043) and at the cervical lymph node (OR: $3.04, P: 0.019)$ after radiotherapy, respectively. Another SNP, $N F-K B I L 1$ rs2071592, was related with a favorable outcome at the cervical lymph node in multiplicative (OR: 0.46, $P$ : 0.011 ) and additive models (OR: 0.53, P: 0.046).

\section{Stratification analyses of the associations between 8 SNPs and the efficacy of radiotherapy}

We performed stratification analyses to evaluate the impact of selected 8 SNPs on the efficacy of radiotherapy in NPC patients sorted by each clinical factor. As shown in Table 5, CDKN2A rs3088440 was significantly associated with the efficacy at the primary tumor and cervical lymph 
Table 2: Genotyping and MAF of 8 SNPs in genes of cell cycle and NF-кB pathways

\begin{tabular}{|c|c|c|c|c|c|}
\hline SNP & Location & $\begin{array}{c}\text { Alleles } \\
\text { (mutant/wild) }\end{array}$ & $\begin{array}{c}\mathbf{N} \\
(\mathbf{A A} / \mathbf{A a} / \\
\mathbf{a a})\end{array}$ & $\begin{array}{c}P \\
(\text { HWE })\end{array}$ & MAF \\
\hline CCND1 rs9344 & Extron & $\mathrm{G} / \mathrm{A}$ & $23 / 86 / 45$ & 0.08 & 0.43 \\
\hline$C D K N 2 A$ rs 3088440 & Downstream 500B & $\mathrm{A} / \mathrm{G}$ & $35 / 80 / 39$ & 0.62 & 0.49 \\
\hline$C D K N 1 A$ rs1059234 & 3'UTR & $\mathrm{C} / \mathrm{T}$ & $2 / 32 / 121$ & 0.94 & 0.12 \\
\hline$I K B K B$ rs 12676482 & Intron & $\mathrm{A} / \mathrm{G}$ & $1 / 34 / 119$ & 0.39 & 0.12 \\
\hline TRAF6 rs4755453 & Upstream 2KB & $\mathrm{C} / \mathrm{G}$ & $2 / 35 / 117$ & 0.73 & 0.13 \\
\hline TNIP1 rs 10036748 & Intron & $\mathrm{C} / \mathrm{T}$ & $8 / 61 / 85$ & 0.49 & 0.25 \\
\hline$N F-K B I L 1$ rs2071592 & Promoter & $\mathrm{T} / \mathrm{A}$ & $37 / 68 / 49$ & 0.17 & 0.46 \\
\hline TRAF6 rs5030437 & Intron & $\mathrm{A} / \mathrm{G}$ & $1 / 33 / 120$ & 0.43 & 0.11 \\
\hline
\end{tabular}

AA/Aa/aa:wild type/heterozygote/mutant homozygote;

Abbreviations: HWE, Hardy-Weinberg equilibrium; MAF, minor allele frequency; UTR, untranslated regions.

Table 3: The associations between 8 SNPs and the efficacy of radiotherapy for the primary tumor

\begin{tabular}{|c|c|c|c|c|c|c|c|c|c|c|c|c|c|}
\hline \multirow{2}{*}{ SNP } & \multirow{2}{*}{ Alleles $^{\mathrm{a}}$} & \multicolumn{2}{|c|}{ MAF } & \multicolumn{2}{|c|}{$\mathbf{N}^{\mathrm{b}}$} & \multicolumn{2}{|c|}{ Multiplicative } & \multicolumn{2}{|l|}{ Additive } & \multicolumn{2}{|l|}{ Dominant } & \multicolumn{2}{|l|}{ Recessive } \\
\hline & & $\begin{array}{c}\text { n- } \\
\text { CR }\end{array}$ & CR & n-CR & CR & OR( $(95 \%$ CI $)$ & $P$ & OR(95\% CI) & $P$ & OR( $(95 \%$ CI $)$ & $P$ & OR( $(95 \%$ CI $)$ & $P$ \\
\hline rs 9344 & $\mathrm{G} / \mathrm{A}$ & 0.38 & 0.44 & $1 / 20 / 8$ & $22 / 66 / 37$ & $0.78(0.43-1.40)$ & 0.400 & $0.82(0.43-1.57)$ & 0.547 & $0.55(0.23-1.32)$ & 0.179 & $0.14(0.02-1.16)$ & 0.069 \\
\hline rs3088440 & $\mathrm{A} / \mathrm{G}$ & 0.19 & 0.10 & $0 / 11 / 18$ & $2 / 20 / 103$ & $2.20(1.01-4.81)$ & 0.043 & $2.37(1.03-5.45)$ & 0.043 & $0.21(0.04-0.96)$ & 0.044 & NA & NA \\
\hline rs1059234 & $\mathrm{C} / \mathrm{T}$ & 0.52 & 0.48 & $8 / 14 / 7$ & $27 / 66 / 32$ & $1.16(0.66-2.06)$ & 0.609 & $1.09(0.60-1.99)$ & 0.781 & $0.64(0.26-1.59)$ & 0.339 & $1.22(0.47-3.19)$ & 0.682 \\
\hline rs 12676482 & $\mathrm{~A} / \mathrm{G}$ & 0.10 & 0.12 & $0 / 6 / 23$ & $1 / 28 / 96$ & $0.85(0.33-2.14)$ & 0.724 & $0.72(0.26-1.96)$ & 0.519 & $1.34(0.52-3.44)$ & 0.542 & NA & NA \\
\hline rs4755453 & $\mathrm{C} / \mathrm{G}$ & 0.12 & 0.13 & $0 / 7 / 22$ & $2 / 28 / 95$ & $0.94(0.39-2.24)$ & 0.880 & $1.14(0.45-2.89)$ & 0.788 & $0.64(0.23-1.78)$ & 0.393 & NA & NA \\
\hline rs 10036748 & $\mathrm{C} / \mathrm{T}$ & 0.29 & 0.24 & $3 / 11 / 15$ & $5 / 50 / 70$ & $1.31(0.70-2.48)$ & 0.400 & $1.25(0.63-2.49)$ & 0.521 & $0.45(0.19-1.06)$ & 0.068 & $2.75(0.52-14.46)$ & 0.234 \\
\hline rs2071592 & $\mathrm{T} / \mathrm{A}$ & 0.31 & 0.50 & $3 / 12 / 14$ & $34 / 56 / 35$ & $0.46(0.25-0.84)$ & 0.011 & $0.53(0.29-0.99)$ & 0.046 & $0.82(0.33-2.00)$ & 0.660 & $0.33(0.09-1.23)$ & 0.099 \\
\hline rs5030437 & $\mathrm{A} / \mathrm{G}$ & 0.14 & 0.11 & $0 / 8 / 21$ & $1 / 25 / 99$ & $1.32(0.57-3.08)$ & 0.518 & $1.52(0.60-3.86)$ & 0.380 & $0.45(0.14-1.42)$ & 0.171 & NA & NA \\
\hline
\end{tabular}

a In the order of mutant/wild; ' In the order of mutant homozygote/heterozygote/wild homozygote;

Abbreviations: CR, complete remission; MAF, minor allele frequency; OR, odds ratio; CI, confidence interval; NA, not applicative. $P$ value $<0.05$ is shown in bold.

Table 4: The associations between 8 SNPs and the efficacy of radiotherapy for the cervical lymph node

\begin{tabular}{|c|c|c|c|c|c|c|c|c|c|c|c|c|c|}
\hline \multirow{2}{*}{ SNP } & \multirow{2}{*}{ Alleles $^{\mathrm{a}}$} & \multicolumn{2}{|c|}{ MAF } & \multicolumn{2}{|c|}{$\mathbf{N}^{\mathbf{b}}$} & \multicolumn{2}{|c|}{ Multiplicative } & \multicolumn{2}{|l|}{ Additive } & \multicolumn{2}{|l|}{ Dominant } & \multicolumn{2}{|l|}{ Recessive } \\
\hline & & $\begin{array}{c}\text { n- } \\
\text { CR }\end{array}$ & CR & n-CR & CR & OR(95\%CI) & $P$ & OR(95\%CI) & $P$ & OR(95\%CI) & $P$ & OR(95\%CI) & $P$ \\
\hline rs9344 & $\mathrm{G} / \mathrm{A}$ & 0.35 & 0.42 & $2 / 8 / 7$ & $14 / 63 / 31$ & $0.75(0.35-1.59)$ & 0.452 & $0.79(0.34-1.82)$ & 0.583 & $0.70(0.23-2.10)$ & 0.523 & $0.87(0.17-4.52)$ & 0.866 \\
\hline rs 3088440 & $\mathrm{~A} / \mathrm{G}$ & 0.21 & 0.08 & $0 / 7 / 10$ & $1 / 15 / 92$ & $3.04(1.15-7.99)$ & 0.019 & $3.58(1.22-10.52)$ & 0.021 & $4.69(1.44-15.3)$ & 0.011 & NA & NA \\
\hline rs 1059234 & $\mathrm{C} / \mathrm{T}$ & 0.53 & 0.50 & $5 / 8 / 4$ & $24 / 61 / 23$ & $1.10(0.54-2.28)$ & 0.788 & $1.03(0.47-2.23)$ & 0.947 & $0.90(0.25-3.21)$ & 0.877 & $1.17(0.35-3.98)$ & 0.797 \\
\hline rs 12676482 & $\mathrm{~A} / \mathrm{G}$ & 0.15 & 0.13 & $0 / 5 / 12$ & $1 / 27 / 80$ & $1.11(0.40-3.10)$ & 0.840 & $1.01(0.32-3.17)$ & 0.992 & $1.05(0.32-3.44)$ & 0.939 & NA & NA \\
\hline rs 4755453 & $\mathrm{C} / \mathrm{G}$ & 0.12 & 0.12 & $1 / 2 / 14$ & $1 / 24 / 83$ & $0.97(0.32-2.99)$ & 0.964 & $1.10(0.34-3.60)$ & 0.875 & $0.76(0.19-3.05)$ & 0.703 & $15.2(0.69-336.20)$ & 0.085 \\
\hline rs 10036748 & $\mathrm{C} / \mathrm{T}$ & 0.29 & 0.26 & $1 / 8 / 8$ & $5 / 46 / 57$ & $1.19(0.54-2.64)$ & 0.668 & $1.13(0.46-2.77)$ & 0.796 & $1.10(0.38-3.17)$ & 0.860 & $1.44(0.14-15.13)$ & 0.764 \\
\hline rs2071592 & $\mathrm{T} / \mathrm{A}$ & 0.29 & 0.46 & $1 / 8 / 8$ & $26 / 47 / 35$ & $0.49(0.22-1.08)$ & 0.073 & $0.49(0.22-1.12)$ & 0.093 & $0.54(0.18-1.64)$ & 0.273 & $0.16(0.02-1.45)$ & 0.104 \\
\hline rs5030437 & $\mathrm{A} / \mathrm{G}$ & 0.06 & 0.12 & $0 / 2 / 15$ & $1 / 24 / 83$ & $0.46(0.10-2.02)$ & 0.290 & $0.45(0.09-2.16)$ & 0.320 & $0.45(0.09-2.21)$ & 0.327 & NA & NA \\
\hline
\end{tabular}

${ }^{a}$ In the order of mutant/wild; 'In the order of mutant homozygote/heterozygote/wild homozygote;

Abbreviations: CR, complete remission; MAF, minor allele frequency; OR, odds ratio; CI, confidence interval; NA, not applicative.

$P$ value $<0.05$ is shown in bold. 
Table 5: Stratification analysis of association between $8 \mathrm{SNPs}$ and the efficacy of radiotherapy in NPC patients

\begin{tabular}{|c|c|c|c|c|c|c|c|c|c|}
\hline \multirow{2}{*}{ SNPs (Allelesa) } & \multirow{2}{*}{ Subgroups (n) } & \multicolumn{2}{|c|}{ Multiplicative } & \multicolumn{2}{|c|}{ Additive } & \multicolumn{2}{|c|}{ Dominant } & \multicolumn{2}{|c|}{ Recessive } \\
\hline & & OR(95\%CI) & $P$ & OR(95\%CI) & $P$ & OR(95\%CI) & $P$ & OR(95\%CI) & $P$ \\
\hline \multicolumn{10}{|c|}{ At the primary tumor } \\
\hline \multirow[t]{4}{*}{ rs3088440(A/G) } & $\geq 51$ y $(80)$ & $4.40(1.58-12.28)$ & 0.003 & $5.04(1.59-16.04)$ & 0.006 & $7.47(2.13-26.17)$ & 0.002 & NA & \\
\hline & High BMI (68) & $4.57(1.41-14.82)$ & 0.007 & $4.50(1.31-15.5)$ & 0.017 & $7.31(1.89-28.23)$ & 0.004 & NA & \\
\hline & Non-smoker (84) & $2.41(0.87-6.67)$ & 0.082 & $2.48(0.86-7.13)$ & 0.092 & $3.18(1.01-10.03)$ & 0.048 & NA & \\
\hline & CRT(122) & $2.11(0.85-5.22)$ & 0.102 & $2.02(0.83-4.93)$ & 0.122 & $2.77(1.01-7.61)$ & 0.049 & NA & \\
\hline \multirow[t]{4}{*}{ rs2071592(T/A) } & $\geq 51$ y $(80)$ & $0.38(0.15-0.92)$ & 0.027 & $0.4(0.17-0.97)$ & 0.042 & $0.50(0.15-1.64)$ & 0.253 & NA & \\
\hline & Low BMI(86) & $2.73(1.12-6.67)$ & 0.024 & $2.75(1.09-6.91)$ & 0.032 & $2.22(0.45-10.89)$ & 0.325 & $4.92(1.43-16.93)$ & 0.012 \\
\hline & Smoker (70) & $3.16(1.16-8.64)$ & 0.020 & $3.20(1.13-9.09)$ & 0.029 & NA & & $2.95(0.77-11.23)$ & 0.113 \\
\hline & CRT(122) & $0.45(0.23-0.87)$ & 0.017 & $0.50(0.26-0.94)$ & 0.031 & $0.40(0.16-1.00)$ & 0.049 & $0.34(0.09-1.23)$ & 0.100 \\
\hline rs5030437 (A/G) & $\geq 51$ y $(80)$ & $2.81(1.01-7.79)$ & 0.040 & $3.07(1.02-9.29)$ & 0.047 & $4.08(1.22-13.68)$ & 0.023 & NA & \\
\hline rs12676482(A/G) & Low BMI(86) & $2.83(0.97-8.21)$ & 0.048 & $3.61(1.05-12.44)$ & 0.042 & $3.61(1.05-12.44)$ & 0.042 & NA & \\
\hline \multicolumn{10}{|c|}{ At the cervical lymph node } \\
\hline \multirow[t]{4}{*}{ rs3088440(A/G) } & $\geq 51$ y $(62)$ & $3.75(1.11-12.68)$ & 0.025 & $4.67(1.22-17.84)$ & 0.024 & $4.67(1.22-17.84)$ & 0.024 & NA & \\
\hline & High BMI (56) & $4.20(0.9-19.69)$ & 0.052 & $3.36(0.77-14.75)$ & 0.108 & $6.6(1.14-38.35)$ & 0.036 & NA & \\
\hline & Non-smoker (67) & $4.42(1.28-15.28)$ & 0.012 & $6.13(1.44-26.05)$ & 0.014 & $6.13(1.44-26.05)$ & 0.014 & NA & \\
\hline & CRT(105) & $3.21(1.12-9.25)$ & 0.024 & $3.25(1.08-9.76)$ & 0.036 & $4.33(1.31-14.37)$ & 0.016 & NA & \\
\hline rs2071592(T/A) & Smoker (58) & $3.97(1.04-15.06)$ & 0.032 & $4.00(1.01-15.85)$ & 0.049 & NA & & $4.33(0.85-22.13)$ & 0.078 \\
\hline
\end{tabular}

${ }^{a}$ In the order of mutant/wild;

Abbreviations: CRT, chemoradiotherapy; BMI, body mass index; OR, odds ratio; CI, confidence interval; NA, not applicable.

High BMI: $\mathrm{BMI} \geq 22.8$; Low BMI: $\mathrm{BMI}<22.8 ; P$ value $<0.05$ is shown in bold.

node among patients $\geq 51$ years old, patients with BMI $\geq 22.8$, non-smoking patients and patients treated with chemoradiotherapy. NF-KBIL1 rs2071592 was related with the efficacy at the primary tumor among patients $\geq$ 51 years old, patients with $\mathrm{BMI}<22.8$, smoking patients and patients treated with chemoradiotherapy, and this SNP was related with the efficacy at the cervical lymph node in smoking patients in multiplicative and additive models. In addition, TRAF6 rs5030437 (among patients $\geq 51$ years old) and $I K B K B$ rs12676482 (among patients with BMI $<22.8$ ) were associated with the efficacy at the primary tumor in multiplicative, additive, and dominant models.

\section{Associations between the 8 SNPs and grade 3-4 acute radiation-induced toxic reactions}

The associations between the selected 8 SNPs and grade 3-4 acute radiation-induced myelosuppression are shown in Table 6. CDKN2A rs3088440 polymorphism was significantly correlated with grade 3-4 acute radiationinduced myelosuppression in multiplicative (OR: 0.21, $P$ : 0.020 ), additive (OR: $0.22, P: 0.045)$ and dominant (OR: $0.21, P: 0.044)$ models after adjustment. Patients carrying the minor A allele of rs3088440 were more resistant to acute radiation-induced myelosuppression. None of the 8 SNPs was found significantly associated with acute radiation-induced dermatitis and oral mucositis after adjustment (Supplementary Tables 3 and 4).

\section{Stratification analyses of the associations between 8 SNPs and grade 3-4 acute radiation- induced toxic reactions}

As shown in Table 7, CCND1 rs9344 was related with grade 3-4 acute radiation-induced oral mucositis in recessive model among patients $<51$ years old, and it was also related with grade 3-4 acute radiation-induced myelosuppression in additive and recessive models among patients with $\mathrm{BMI}<22.8$. CDKN2A rs3088440 polymorphism was correlated with grade 3-4 acute radiation-induced myelosuppression among III-IV stage patients and patients treated with chemoradiotherapy. $I K B K B$ rs 12676482 was associated with grade 3-4 acute radiation-induced myelosuppression in multiplicative and additive models among non-smoking patients. Individuals carrying the minor A allele of rs9344 and rs3088440 were less sensitive to the radiation-induced toxicity, while patients with the minor A allele of rs12676482 had a higher risk of grade 3-4 acute radiation-induced myelosuppression, compared with the G allele.

\section{DISCUSSION}

Deregulation of the cell cycle is one of the most frequent alterations in the process of tumor development, and genetic polymorphisms of genes involved in cell 
Table 6: The associations between 8 SNPs and the grade 3-4 acute radiation-induced myelosuppression

\begin{tabular}{|c|c|c|c|c|c|c|c|c|c|c|c|c|c|}
\hline \multirow{2}{*}{ SNP } & \multirow{2}{*}{ Alleles ${ }^{a}$} & \multicolumn{2}{|c|}{ MAF } & \multicolumn{2}{|c|}{$\mathbf{N}^{\mathbf{b}}$} & \multicolumn{2}{|c|}{ Multiplicative } & \multicolumn{2}{|l|}{ Additive } & \multicolumn{2}{|l|}{ Dominant } & \multicolumn{2}{|l|}{ Recessive } \\
\hline & & G3+ & G0-2 & G3+ & G0-2 & OR(95\% CI) & $P$ & OR( $(95 \% \mathrm{CI})$ & $P$ & OR( $(95 \% \mathrm{CI})$ & $P$ & OR( $(95 \% \mathrm{CI})$ & $P$ \\
\hline rs9344 & $\mathrm{G} / \mathrm{A}$ & 0.38 & 0.44 & $5 / 14 / 13$ & $18 / 72 / 32$ & $0.76(0.43-1.33)$ & 0.331 & $0.74(0.39-1.39)$ & 0.347 & $0.55(0.23-1.32)$ & 0.179 & $0.99(0.32-3.05)$ & 0.982 \\
\hline rs3088440 & $\mathrm{A} / \mathrm{G}$ & 0.03 & 0.14 & $0 / 2 / 30$ & $2 / 29 / 91$ & $0.21(0.05-0.88)$ & 0.020 & $0.22(0.05-0.97)$ & 0.045 & $0.21(0.04-0.96)$ & 0.044 & NA & NA \\
\hline rs1059234 & $\mathrm{C} / \mathrm{T}$ & 0.47 & 0.49 & $8 / 14 / 10$ & $27 / 66 / 29$ & $0.91(0.53-1.58)$ & 0.743 & $0.81(0.45-1.45)$ & 0.473 & $0.64(0.26-1.59)$ & 0.339 & $0.9(0.34-2.38)$ & 0.840 \\
\hline rs 12676482 & $\mathrm{~A} / \mathrm{G}$ & 0.16 & 0.11 & $1 / 8 / 23$ & 0/26/96 & $1.55(0.71-3.41)$ & 0.271 & $1.55(0.64-3.77)$ & 0.330 & $1.34(0.52-3.44)$ & 0.542 & NA & NA \\
\hline rs 4755453 & $\mathrm{C} / \mathrm{G}$ & 0.09 & 0.14 & $0 / 6 / 26$ & $2 / 29 / 91$ & $0.66(0.26-1.66)$ & 0.374 & $0.60(0.23-1.56)$ & 0.300 & $0.64(0.23-1.78)$ & 0.393 & NA & NA \\
\hline rs 10036748 & $\mathrm{C} / \mathrm{T}$ & 0.17 & 0.27 & $1 / 9 / 22$ & $7 / 52 / 63$ & $0.56(0.28-1.14)$ & 0.105 & $0.52(0.24-1.16)$ & 0.112 & $0.45(0.19-1.06)$ & 0.068 & $1.28(0.12-13.79)$ & 0.840 \\
\hline rs2071592 & $\mathrm{T} / \mathrm{A}$ & 0.48 & 0.45 & $10 / 11 / 11$ & $27 / 57 / 38$ & $1.13(0.65-1.95)$ & 0.674 & $0.99(0.57-1.73)$ & 0.982 & $0.82(0.33-2.00)$ & 0.660 & $1.22(0.48-3.1)$ & 0.672 \\
\hline rs5030437 & $\mathrm{A} / \mathrm{G}$ & 0.06 & 0.13 & $0 / 4 / 28$ & $1 / 29 / 92$ & $0.46(0.16-1.35)$ & 0.148 & $0.44(0.14-1.36)$ & 0.156 & $0.45(0.14-1.42)$ & 0.171 & NA & NA \\
\hline
\end{tabular}

${ }^{a}$ In the order of mutant/wild; 'In the order of mutant homozygote/heterozygote/wild homozygote;

Abbreviations: CR, complete remission; MAF, minor allele frequency; G, grade; OR, odds ratio; CI, confidence interval; NA, not applicative. $P$ value $<0.05$ is shown in bold.

Table 7: Stratification analysis of association between SNPs and the acute radiation-induced toxic reactions in NPC patients

\begin{tabular}{|c|c|c|c|c|c|c|c|c|c|}
\hline \multirow{2}{*}{ SNPs (Alleles ${ }^{\text {a }}$ ) } & \multirow{2}{*}{$\begin{array}{c}\text { Subgroups } \\
\text { (n) }\end{array}$} & \multicolumn{2}{|c|}{ Multiplicative } & \multicolumn{2}{|c|}{ Additive } & \multicolumn{2}{|c|}{ Dominant } & \multicolumn{2}{|c|}{ Recessive } \\
\hline & & OR(95\%CI) & $P$ & OR(95\%CI) & $P$ & OR(95\%CI) & $\boldsymbol{P}$ & OR(95\%CI) & $P$ \\
\hline \multicolumn{10}{|c|}{ Grade 3-4 oral mucositis } \\
\hline rs9344 (G/A) & $<51 \mathrm{y}(74)$ & $\begin{array}{c}0.65(0.33- \\
1.27)\end{array}$ & 0.208 & $\begin{array}{c}0.65(0.33- \\
1.28)\end{array}$ & 0.211 & $\begin{array}{c}1.15(0.45- \\
2.96)\end{array}$ & 0.766 & $\begin{array}{c}0.08(0.01- \\
0.66)\end{array}$ & 0.019 \\
\hline \multicolumn{10}{|c|}{ Grade 3-4 myelosuppression } \\
\hline \multirow[t]{2}{*}{ rs3088440 (A/G) } & $\begin{array}{c}\text { III-IV } \\
\text { stage(131) }\end{array}$ & $\begin{array}{c}0.24(0.06- \\
1.06)\end{array}$ & 0.043 & $\begin{array}{c}0.25(0.06- \\
1.09)\end{array}$ & 0.065 & $\begin{array}{c}0.24(0.05- \\
1.09)\end{array}$ & 0.064 & NA & \\
\hline & CRT(122) & $\begin{array}{c}0.23(0.05- \\
1.01)\end{array}$ & 0.035 & $\begin{array}{c}0.24(0.06- \\
1.05)\end{array}$ & 0.058 & $\begin{array}{c}0.23(0.05- \\
1.04)\end{array}$ & 0.057 & NA & \\
\hline rs9344 (G/A) & $\begin{array}{c}\text { Low } \\
\text { BMI(86) }\end{array}$ & $\begin{array}{c}0.49(0.23- \\
1.05)\end{array}$ & 0.064 & $\begin{array}{c}0.42(0.18- \\
1.00)\end{array}$ & 0.049 & $\begin{array}{c}0.29(0.1- \\
0.86)\end{array}$ & 0.026 & NA & \\
\hline rs12676482(A/G) & $\begin{array}{c}\text { Non- } \\
\text { smoker(84) }\end{array}$ & $\begin{array}{c}2.57(1.01- \\
6.52)\end{array}$ & 0.042 & $\begin{array}{c}2.83(1.03- \\
7.77)\end{array}$ & 0.043 & $\begin{array}{c}2.65(0.89- \\
7.85)\end{array}$ & 0.079 & NA & \\
\hline
\end{tabular}

${ }^{\text {a }}$ In the order of mutant/wild;

Abbreviations: CRT, chemoradiotherapy; BMI, body mass index; OR, odds ratio; CI, confidence interval; NA, not applicable.

High BMI: BMI $\geq 22.8$; Low BMI: $\mathrm{BMI}<22.8$; $P$ value $<0.05$ is shown in bold.

cycle control have been implicated in development and prognosis of many cancers. The NF- $\kappa \mathrm{B}$ signaling pathway has also been linked with several cancers by regulating transcription of its downstream target genes, including genes involved in cell cycle pathway [28, 40]. Many studies have found that NF- $\kappa \mathrm{B}$ signaling was exceedingly activated and some target genes of $\mathrm{NF}-\kappa \mathrm{B}$ were upregulated in several cancers [41-43]. As far as we know, this is the first time to investigate the impact of SNPs in the cell cycle pathway and $\mathrm{NF}-\kappa \mathrm{B}$ signaling pathway genes on the efficacy and toxicity of radiotherapy in NPC patients treated with radiotherapy (or chemoradiotherapy). Our results showed that 4 SNPs (CDKN2A rs3088440,
NF-KBIL1 rs2071592, TRAF6 rs5030437 and IKBKB rs12676482) were related with the efficacy of radiotherapy and 3 SNPs (CDKN2A rs3088440, CCND1 rs9344 and $I K B K B$ rs 12676482) were related with the acute radiationinduced toxic reactions in NPC patients.

The results of logistic regressions in whole patients and some subgroups indicated that CDKN2A rs3088440 polymorphism was markedly associated with a poorer efficacy of radiotherapy at the primary tumor and cervical lymph node, as well with a decreased risk of grade 3-4 acute radiation-induced myelosuppression. This revealed that the mutant A allele of rs3088440 was related with a more robust resistance to radiotherapy than 
the $\mathrm{G}$ allele. Rs3088440 was identified in the 3'-UTR of $C D K N 2 A$ and has been reported to be relevant to the susceptibility and prognosis of several cancers in spite of inconsistent results in previous studies [44-46]. The association between rs3088440 polymorphism and the increased risk of HPV16-positive oropharynx cancer has also been reported, while no significant prognostic effect of rs3088440 polymorphism was found on the survival after definitive chemoradiotherapy in HPV16-positive oropharynx cancer patients [45]. Two previous studies have reported the significant relation between the A allele of $C D K N 2 A$ rs3088440 polymorphism and the higher risk of melanoma $[46,47]$, but other studies could not replicate such result $[48,49]$. In another separate study, CDKN2A rs3088440 polymorphism was associated with reduced risk of progression from Barrett's esophagus to esophageal adenocarcinoma [50]. The result of their in vitro functional studies suggested that the transcript of CDKN2A may be suppressed by miR-663b in a rs3088440 G allele-dependent manner. Under that circumstance, the rs3088440 A mutant would potentially impair the binding of miR-663b to the CDKN2A 3' UTR and lead to enhanced expression of the $\mathrm{p} 14 / \mathrm{p} 16$ proteins. The results of the present study suggested a potential mechanism that the enhancement of $C D K N 2 A$ expression due to the $\mathrm{G}>\mathrm{A}$ substitution results in the proliferation of NPC cells, which ulteriorly weakens the treatment effect of radiotherapy and alleviates the radiation-induced toxicity.

We also found that the AA genotype of CCND1 rs9344 was associated with a decreased risk of grade 3-4 oral mucositis among patients $<51$ years old. And among patients with BMI $<22.8$, this SNP was related with grade 3-4 myelosuppression in multiplicative (OR: 0.42, $P: 0.049$ ) and additive (OR: 0.29, P: 0.026) models. The $\mathrm{G}>\mathrm{A}$ mutant of $C C N D 1$ rs9344 changes the splicing of exon 4 and then alters the CCND1 expression, which may promote its oncogenic potential [51]. Previous studies mostly investigated the impact of rs9344 on the susceptibility and survival of many cancers, such as breast cancer, NPC, HCC and oral cancer [21, 52, 53]. However, there has been no study exploring the influence of this SNP on the toxicity of radiotherapy. According to this present study, the A allele of CCND1 rs9344 was associated with a more robust resistance to radiotherapy and the result needs more large-size studies to duplicate.

In addition, $N F-K B I L 1$ rs2071592 was related with a more favorable outcome at the primary tumor in the multivariate logistic regression as well as among patients $\geq 51$ years old and treated with chemoradiotherapy. But on the contrary, rs2071592 was linked with a poorer outcome at the primary tumor among patients with BMI < 22.8 and smoking patients. NF-KBIL1 is a novel inhibitor of NF- $\kappa \mathrm{B}$ signaling and the polymorphism rs2071592 in its gene promoter region has been reported to be significantly associated with rheumatoid arthritis (RA) [54] and systemic lupus erythematosus (SLE) [34]. The
A allele of this SNP is presumed to disrupt the binding of transcriptional factor and affect the expression of $\mathrm{NF}$ $K B I L 1$. Since our contradictory results, the authentic relationship between NF-KBIL1 rs2071592 with the efficacy of radiotherapy in NPC remains uncertain and the exact mechanism needs more studies to explain.

In the subgroup analysis, TRAF6 rs5030437 and $I K B K B$ rs 12676482 were associated with less favorable outcome at the primary tumor in patients $\geqq 51$ years old and patients with BMI $<22.8$, respectively. TRAF6 and IKBKB are positive regulators of $\mathrm{NF}-\kappa \mathrm{B}$ signaling, of which rs5030437 and rs12676482 are both identified in the intron region of corresponding gene. These two SNPs may upregulate the gene expression by affecting mRNA splicing, localization or stability, and reinforce the NF- $\kappa B$ signaling, which facilitates the proliferation of NPC cells and weakens the effect of radiotherapy [55]. Another possible mechanism is that the rs5030437 or rs12676482 is in linkage disequilibrium (LD) with a certain functional variant that really mediates this process [56]. Whatever, there has no report about the correlation between rs5030437 and rs12676482 polymorphisms with the prognosis of NPC, and more studies are requisite to verify the specific mechanism.

The obvious advantage of our study lies in that this is the first time to assess the association of SNPs in cell cycle pathway and NF- $\kappa \mathrm{B}$ signaling pathway genes with clinical responses to radiotherapy in NPC patients. However, our study still had some limitations. First, the small sample size reduced the statistical power, especially the limited numbers of patients in some subgroups, which resulted in the inconsistent results for certain findings in different subgroups. Second, the mechanisms that how these SNPS influenced the treatment efficacy and toxicity of radiotherapy in NPC patients were not fully understood, as those were beyond the scope of the present study. Third, we did not detect the levels of corresponding mRNA and protein expression of each studied gene, so the findings in this study still remain to be confirmed in more researches.

\section{MATERIALS AND METHODS}

\section{Study population}

This study collected 154 NPC patients treated with radiotherapy (with or without joint chemotherapy) from the Jiangxi Cancer Hospital from April 2014 to September 2015. The patients were eligible for this study if they met the following criteria: pathologically confirmed NPC; no distant metastasis; performance status score $\leq 1$; no severe dysfunction of lung, heart, liver and kidney; no treatment with any anticancer therapies before radiotherapy; and signing the informed consent before this study. Exclusion criteria included: pregnancy or lactation; active infection before radiotherapy; and previous or concomitant malignancies. We recorded all demographics 
and clinic pathologic data of 154 patients including age, gender, body mass index (BMI), smoking and drinking status, TNM ( $7^{\text {th }}$ UICC) stage and with/without chemotherapy. The study was approved by the Ethics Committee of Cancer Hospital of Jiangxi Province (China) and we applied for clinical admission for this study through the Chinese Clinical Trial Registry (www.chictr. org.cn, registration number: ChiCTR-OPC-14005257).

\section{Efficacy regimen}

All selected patients were treated with IMRT technique. Most patients (122) were treated with IMRT as well as chemotherapy while the other 32 were treated with IMRT alone. For nasopharyngeal primary focus and cervical positive lymph nodes, the total dose of radiotherapy was 66-70 Gy in 30-33 fractions, and for cervical drainage region, patients received 54-60 Gy in all in 30-33 fractions. One fraction was administrated daily and five fractions per week. The inductive chemotherapy and concurrent chemotherapy referred to platinum-based chemotherapy regimens.

\section{Evaluation on the radiotherapy treatment efficacy and toxic reactions}

The curative efficacy at the primary tumor and cervical lymph node was evaluated by magnetic resonance imaging (MRI) directly after finishing radiotherapy. According to the Response Evaluation Criteria In Solid Tumors (RECIST) of World Health Organization (WHO), the efficacy was defined as complete remission (CR), partial remission $(\mathrm{PR})$, stable disease $(\mathrm{SD})$ or progression disease (PD) based on the tumor volume. All patients of this study were subdivided into two groups: $\mathrm{CR}$, and non-CR consisting of PR, SD and PD for the efficacy of primary tumor and cervical lymph node.

We observed and recorded acute radiation-induced toxic reactions, including myelosuppression, dermatitis and oral mucositis, once a day from the first day to the end of radiotherapy. All toxic reactions were evaluated and classified as grade $0-4$ based on the acute radiation toxicity grading criterion of the Radiation Therapy Oncology Group or European Organization for Research and Treatment of Cancer (RTOG/EORTC). According to the grading results, we divided the patients into two groups: non-sensitive or mildly radiosensitive (grade $0-2$ ), and highly radiosensitive (grade 3-4).

\section{DNA extraction and genotyping}

Venous blood sample $(3 \mathrm{~mL})$ was taken from each patient before radiotherapy and stored in EDTA anticoagulated tubes at $-20^{\circ} \mathrm{C}$. Genomic DNA was extracted using Wizard Genomic DNA Purification Kit (Promega, Madison, USA). Genotyping for 8 SNPs was performed by Sequenom MassARRAY system (Sequenom, San Diego, California, USA). Primers were designed by using Assay Designer 3.1 and the polymerase chain reaction (PCR) was performed by following scheme: an initial denaturation for $15 \mathrm{~min}$ at $94^{\circ} \mathrm{C}$, then 45 cycles of $20 \mathrm{~s}$ at $94^{\circ} \mathrm{C}, 30 \mathrm{~s}$ at $56^{\circ} \mathrm{C}$, and $1 \mathrm{~min}$ at $72^{\circ} \mathrm{C}$, and a final elongation for $3 \mathrm{~min}$ at $72^{\circ} \mathrm{C}$. The products of PCR were purified using shrimp alkaline phosphatase (SAP) according to following process: initial incubation for 40 min at $37^{\circ} \mathrm{C}$, followed by $85^{\circ} \mathrm{C}$ for $5 \mathrm{~min}$. The purified products were extended by reactions using extension primers and subsequently desalted by the addition of a cation exchange resin. The desalinated products were transferred in CHIP after being centrifuged at $4000 \mathrm{rpm}$ for $4 \mathrm{~min}$. Data from CHIP were analyzed by Typer Analyzer.

\section{Statistical analysis}

The association of each clinical factor with the efficacy or toxic reactions after radiotherapy was computed using chi-square test or t-test. Multivariate logistic regression was carried out to determine the associations between each SNP and non-CR of primary tumor and cervical lymph node as well as grade 3-4 toxic reactions, with $\mathrm{CR}$ and grade $0-2$ as the reference, respectively. The odds ratio (OR) and the corresponding 95\% confidence interval (CI) were computed after adjustment for age, gender, BMI, smoking and drinking habit, carcinoma stage, and with/without chemotherapy. All logistic regressions were conducted by four models: multiplicative, additive, dominant and recessive models, which were performed by PLINK 1.07 (https://www.coggenomics.org/plink2). If $\mathrm{X}$ is the major allele and $\mathrm{x}$ is the minor allele, the analysis in multiplicative model breaks down the genotypes to compare the total numbers of $\mathrm{X}$ and $x$ alleles of each SNP in two groups, regardless of the genotypes. The additive model is for the additive effect of each SNP, with a direction of the regression coefficient that represents the effect of each extra $\mathrm{x}$ allele. Dominant and recessive models are both tests for the minor allele with two of the three genotypes pooled. The dominant model means $(\mathrm{xx}+\mathrm{Xx})$ versus $\mathrm{XX}$ and the recessive model means $\mathrm{xx}$ versus $(\mathrm{XX}+\mathrm{Xx})$ [57]. In addition, stratification analyses were performed to determine the associations of SNPs and radiotherapy efficacy and toxic reactions in many subgroups sorted by age, gender, BMI, smoking and drinking habit, carcinoma stage, and with/without chemotherapy. The difference was considered statistically significant if $P$ value $<0.05$.

\section{Abbreviations}

NPC: nasopharyngeal carcinoma; IMRT: intensity modulated radiotherapy; CR: complete remission; PR: partial remission; SD: stable disease; PD: progression 
disease; SNP: single nucleotide polymorphism; CDK: cyclin-dependent kinase; CDKN: CDK inhibitor; CCND1: cyclin D1; NF-кB: nuclear factor-kappa B; HNSCC: head and neck squamous cell carcinoma; RANK: receptor activator of NF- $\mathrm{BB}$; TNFAIP3: tumor necrosis factor alpha-induced protein 3; NFKBIA: NF- $\kappa$ B inhibitor alpha: CYLD: cylindromatosis; TRAF6: tumor necrosis factor receptor-associated factor-6; TNIP1: TNFAIP3 interacting protein 1 ; I $\mathrm{B}$ : inhibitor of $\kappa \mathrm{B}$; IKK: inhibitor of $\kappa \mathrm{B}$ kinase; NF-KBIL1: NF- $\kappa \mathrm{B}$ inhibitor-like protein 1; MRI: magnetic resonance imaging; RECIST: response evaluation criteria in solid tumors; RTOG: radiation therapy oncology group; EORTC: European organization for research and efficacy of cancer.

\section{CONFLICTS OF INTEREST}

The authors claim that no conflicts of interest exist.

\section{FUNDING}

This research was supported by the National Natural Science Foundation of China (81460409 and 81301924), the New Xiangya Talent Project of the Third Xiangya Hospital of Central South University (JY201505), and the Independent Exploration and Innovation Project for Postgraduate of Central South University (2016zzts564).

\section{Author contributions}

JL designed the studies; LL, XG, LH and GY performed the experiments; $\mathrm{CG}, \mathrm{JY}$ and $\mathrm{YH}$ analyzed the data; YL, MH and CJ collected tumor specimens and clinical data; $\mathrm{CG}, \mathrm{YH}$ and JY wrote the manuscript. All authors have read and approved the final manuscript.

\section{REFERENCES}

1. Siegel RL, Miller KD, Jemal A. Cancer statistics, 2016. CA Cancer J Clin. 2016; 66:7-30.

2. Kamran SC, Riaz N, Lee N. Nasopharyngeal carcinoma. Surgical oncology clinics of North America. 2015; 24:547-561

3. Wang M, Tian H, Li G, Ge T, Liu Y, Cui J, Han F. Significant benefits of adding neoadjuvant chemotherapy before concurrent chemoradiotherapy for locoregionally advanced nasopharyngeal carcinoma: a meta-analysis of randomized controlled trials. Oncotarget. 2016; 7:4837548390. doi: 10.18632/oncotarget.10237.

4. Wang W, Feng M, Fan Z, Li J, Lang J. Clinical outcomes and prognostic factors of 695 nasopharyngeal carcinoma patients treated with intensity-modulated radiotherapy. Biomed Res Int. 2014; 2014:814948.

5. Trotti A. Toxicity in head and neck cancer: a review of trends and issues. International journal of radiation oncology, biology, physics. 2000; 47:1-12.
6. Beetz I, Schilstra C, Burlage FR, Koken PW, Doornaert P, Bijl HP, Chouvalova O, Leemans CR, de Bock GH, Christianen ME, van der Laan BF, Vissink A, Steenbakkers RJ, et al. Development of NTCP models for head and neck cancer patients treated with three-dimensional conformal radiotherapy for xerostomia and sticky saliva: the role of dosimetric and clinical factors. Radiother Oncol. 2012; 105:86-93.

7. Li SS, Tang QL, Wang SH, Wang S, Yang XM. Simultaneously targeting bcl-2 and Akt pathways sensitizes nasopharyngeal carcinoma to tumor necrosis factorrelated apoptosis-inducing ligand. Cancer biotherapy \& radiopharmaceuticals. 2012; 27:88-95.

8. Kerns SL, Ostrer H, Rosenstein BS. Radiogenomics: using genetics to identify cancer patients at risk for development of adverse effects following radiotherapy. Cancer discovery. 2014; 4:155-165.

9. Zou Y, Song T, Yu W, Zhao R, Wang Y, Xie R, Chen T, $\mathrm{Wu}$ B, Wu S. XRCC3 polymorphisms are associated with the risk of developing radiation-induced late xerostomia in nasopharyngeal carcinoma patients treated with intensity modulation radiated therapy. Japanese Journal of Clinical Oncology. 2014; 44:241-248.

10. Guo Z, Shu Y, Zhou H, Zhang W, Wang H. Radiogenomics helps to achieve personalized therapy by evaluating patient responses to radiation treatment. Carcinogenesis. 2015; 36:307-317.

11. Wang YP, Lin CF, Tsai SC, Tsai CH, Yeh TH. Upregulation of caveolin-1 correlate with Akt expression and poor prognosis in NPC patients. The Laryngoscope. 2015; 125:E231-238.

12. Ghazali N, Shaw RJ, Rogers SN, Risk JM. Genomic determinants of normal tissue toxicity after radiotherapy for head and neck malignancy: A systematic review. Oral Oncology. 2012; 48:1090-1100.

13. Cheuk IW, Yip SP, Kwong DL, Wu VW. Association of XRCC1 and XRCC3 gene haplotypes with the development of radiation-induced fibrosis in patients with nasopharyngeal carcinoma. Mol Clin Oncol. 2014; 2:553-558.

14. Li H, You Y, Lin C, Zheng M, Hong C, Chen J, Li D, Au WW, Chen Z. XRCC1 codon 399Gln polymorphism is associated with radiotherapy-induced acute dermatitis and mucositis in nasopharyngeal carcinoma patients. Radiation oncology. 2013; 8:31.

15. Karrman K, Castor A, Behrendtz M, Forestier E, Olsson L, Ehinger M, Biloglav A, Fioretos T, Paulsson K, Johansson B. Deep sequencing and SNP array analyses of pediatric T-cell acute lymphoblastic leukemia reveal NOTCH1 mutations in minor subclones and a high incidence of uniparental isodisomies affecting CDKN2A. J Hematol Oncol. 2015; 8:42.

16. Kim WB, Han HJ, Lee HJ, Park SS, Song TJ, Kim HK, Suh SO, Kim YC, Choi SY. Expression and clinical significance of cell cycle regulatory proteins in gallbladder and extrahepatic bile duct cancer. Ann Surg Oncol. 2009; 16:23-34. 
17. Qin LY, Zhao LG, Chen X, Li P, Yang Z, Mo WN. The CCND1 G870A gene polymorphism and brain tumor risk: a meta-analysis. Asian Pac J Cancer Prev. 2014; 15:3607-3612.

18. Absenger G, Benhaim L, Szkandera J, Zhang W, Yang D, Labonte MJ, Pichler M, Stotz M, Samonigg H, Renner W, Gerger A, Lenz HJ. The cyclin D1 (CCND1) rs9344 G>A polymorphism predicts clinical outcome in colon cancer patients treated with adjuvant 5-FU-based chemotherapy. Pharmacogenomics J. 2014; 14:130-134.

19. Tang W, Yu P, Wang Y, Kang M, Sun B, Yin J, Gu H. Lack of association between cyclin D1 A870G (rs9344) polymorphism and esophageal squamous cell carcinoma risk: case-control study and meta-analysis. Int J Clin Exp Med. 2015; 8:12685-12695.

20. Wang N, Qian X, Wang S, Gao H, Wang L, Huo Y, Zhang S. CCND1 rs9344 polymorphisms are associated with the genetic susceptibility to cervical cancer in Chinese population. Mol Carcinog. 2012; 51:196-205.

21. Shih LC, Tsai CW, Tsai MH, Tsou YA, Chang WS, Li FJ, Lee MH, Bau DT. Association of cyclin D1 genotypes with nasopharyngeal carcinoma risk. Anticancer Res. 2012; 32:1093-1098.

22. Kibel AS, Suarez BK, Belani J, Oh J, Webster R, BrophyEbbers M, Guo C, Catalona WJ, Picus J, Goodfellow PJ. CDKN1A and CDKN1B polymorphisms and risk of advanced prostate carcinoma. Cancer Res. 2003; 63:2033-2036.

23. Li J, Li Z, Kan Q, Sun S, Li Y, Wang S. Association of p21 3' UTR gene polymorphism with cancer risk: Evidence from a meta-analysis. Sci Rep. 2015; 5:13189.

24. Tripathi BA, Banerjee S, Chunder N, Roy A, Sengupta A, Roy B, Roychowdhury S, Panda CK. Differential alterations of the genes in the CDKN2A-CCND1-CDK4-RB1 pathway are associated with the development of head and neck squamous cell carcinoma in Indian patients. J Cancer Res Clin Oncol. 2003; 129:642-650.

25. Larque AB, Conde L, Hakim S, Alos L, Jares P, Vilaseca I, Cardesa A, Nadal A. P16(INK(4)a) overexpression is associated with CDKN2A mutation and worse prognosis in HPV-negative laryngeal squamous cell carcinomas. Virchows Arch. 2015; 466:375-382.

26. Vargas-Torres SL, Portari EA, Klumb EM, Guillobel HC, de Camargo MJ, Russomano FB, Macedo JM. Association of CDKN2A polymorphisms with the severity of cervical neoplasia in a Brazilian population. Biomarkers. 2014; 19:121-127.

27. Boughton B. BRAF and CDKN2A mutations in secondary high-grade glioma. Lancet Oncol. 2015; 16:e201.

28. Rinkenbaugh AL, Baldwin AS. The NF-kappaB Pathway and Cancer Stem Cells. Cells. 2016; 5.

29. Otto C, Giefing M, Massow A, Vater I, Gesk S, Schlesner M, Richter J, Klapper W, Hansmann ML, Siebert R, Kuppers R. Genetic lesions of the TRAF3 and MAP3K14 genes in classical Hodgkin lymphoma. British journal of haematology. 2012; 157:702-708.

30. Ruebner M, Lux MP, Renner SP, Schulz-Wendtland R, Ekici AB, Hartmann A, Beckmann MW, Fasching PA, Ungerback J, Belenki D, Jawad ul-Hassan A, Fredrikson $\mathrm{M}$, Fransen K, et al. Genetic variation and alterations of genes involved in NFkappaB/TNFAIP3- and NLRP3inflammasome signaling affect susceptibility and outcome of colorectal cancer. Biomed Res Int. 2012; 33:2126-2134.

31. Hein A, Bayer CM, Schrauder MG, Haberle L, Heusinger $\mathrm{K}$, Strick R. Polymorphisms in the RANK/RANKL genes and their effect on bone specific prognosis in breast cancer patients. 2014; 2014:842452.

32. Zheng H, Dai W, Cheung AK, Ko JM, Kan R, Wong BW, Leong MM, Deng M, Kwok TC, Chan JY, Kwong DL, Lee $\mathrm{AW}, \mathrm{Ng} \mathrm{WT}$, et al. Whole-exome sequencing identifies multiple loss-of-function mutations of NF-kappaB pathway regulators in nasopharyngeal carcinoma. Proceedings of the National Academy of Sciences of the United States of America. 2016; 113:11283-11288.

33. Carlotti F, Dower SK, Qwarnstrom EE. Dynamic shuttling of nuclear factor kappa B between the nucleus and cytoplasm as a consequence of inhibitor dissociation. The Journal of biological chemistry. 2000; 275:41028-41034.

34. Cen H, Zhou M, Leng RX, Wang W, Feng CC, Li BZ, Zhu Y, Yang XK, Yang M, Zhai Y, Zhang M, Hu LF, Li R, et al. Genetic interaction between genes involved in NF-kappaB signaling pathway in systemic lupus erythematosus. Molecular immunology. 2013; 56:643-648.

35. Gao L, Coope H, Grant S, Ma A, Ley SC, Harhaj EW. ABIN1 protein cooperates with TAX1BP1 and A20 proteins to inhibit antiviral signaling. The Journal of biological chemistry. 2011; 286:36592-36602.

36. Byrne JC, Ni Gabhann J, Lazzari E, Mahony R, Smith S, Stacey K, Wynne C, Jefferies CA. Genetics of SLE: functional relevance for monocytes/macrophages in disease. Clinical \& developmental immunology. 2012; 2012:582352.

37. Song Z, Yao C, Yin J, Tong C, Zhu D, Sun Z, Jiang J, Shao M, Zhang Y, Deng Z, Tao Z, Sun S, Bai C. Genetic variation in the TNF receptor-associated factor 6 gene is associated with susceptibility to sepsis-induced acute lung injury. Journal of translational medicine. 2012; 10:166.

38. Wadley AL, Hendry LM, Kamerman PR, Chew CS, Price P, Cherry CL, Lombard Z. Role of TNF block genetic variants in HIV-associated sensory neuropathy in black Southern Africans. European journal of human genetics: EJHG. $2015 ; 23: 363-368$.

39. Li Y, Wu Z, Zhang S, Chen S, Li P, Li J, Cao C, Liu B, Zhang F, Li Y. Genetic variants of IkappaB Kinase beta (IKBKB) and Polymerase beta (POLB) were not associated with systemic lupus erythematosus risk in a Chinese Han population. PLoS One. 2015; 10:e132556. 
40. Stein SJ, Baldwin AS. NF-kappaB suppresses ROS levels in $\mathrm{BCR}-\mathrm{ABL}(+)$ cells to prevent activation of JNK and cell death. Oncogene. 2011; 30:4557-4566.

41. Bang D, Wilson W, Ryan M, Yeh JJ, Baldwin AS. GSK3alpha promotes oncogenic KRAS function in pancreatic cancer via TAK1-TAB stabilization and regulation of noncanonical NF-kappaB. Cancer Discov. 2013; 3:690-703.

42. Yu J, Wang Y, Yan F, Zhang P, Li H, Zhao H, Yan C, Yan F, Ren X. Noncanonical NF-kappaB activation mediates STAT3-stimulated IDO upregulation in myeloid-derived suppressor cells in breast cancer. Journal of immunology (Baltimore, Md: 1950). 2014; 193:2574-2586.

43. Hayden MS, Ghosh S. Shared principles in NF-kappaB signaling. Cell. 2008; 132:344-362.

44. Zhang Y, Sturgis EM, Zafereo ME, Wei Q, Li G. p14ARF genetic polymorphisms and susceptibility to second primary malignancy in patients with index squamous cell carcinoma of the head and neck. Cancer. 2011; 117:1227-1235.

45. Song X, Sturgis EM, Huang Z, Li X, Li C, Wei Q, Li G. Potentially functional variants of p14ARF are associated with HPV-positive oropharyngeal cancer patients and survival after definitive chemoradiotherapy. Carcinogenesis. 2014; 35:62-68

46. Kumar R, Smeds J, Berggren P, Straume O, Rozell BL, Akslen LA, Hemminki K. A single nucleotide polymorphism in the 3'untranslated region of the CDKN2A gene is common in sporadic primary melanomas but mutations in the CDKN2B, CDKN2C, CDK4 and p53 genes are rare. Int J Cancer. 2001; 95:388-393.

47. Maccioni L, Rachakonda PS, Bermejo JL, Planelles D, Requena C, Hemminki K, Nagore E, Kumar R. Variants at the 9p21 locus and melanoma risk. BMC Cancer. 2013; $13: 325$.

48. Debniak T, Scott RJ, Huzarski T, Byrski T, Rozmiarek A, Debniak B, Zaluga E, Maleszka R, Kladny J, Gorski B, Cybulski C, Gronwald J, Kurzawski G, et al. CDKN2A common variants and their association with melanoma risk: a population-based study. Cancer Res. 2005; 65:835-839.

49. Ibarrola-Villava M, Fernandez LP, Pita G, Bravo J, Floristan U, Sendagorta E, Feito M, Aviles JA, MartinGonzalez M, Lazaro P, Benitez J, Ribas G. Genetic analysis of three important genes in pigmentation and melanoma susceptibility: CDKN2A, MC1R and HERC2/OCA2. Exp Dermatol. 2010; 19:836-844.

50. Buas MF, Levine DM, Makar KW, Utsugi H, Onstad L, Li X, Galipeau PC, Shaheen NJ, Hardie LJ, Romero Y, Bernstein L, Gammon MD, Casson AG, et al. Integrative post-genome-wide association analysis of CDKN2A and TP53 SNPs and risk of esophageal adenocarcinoma. Carcinogenesis. 2014; 35:2740-2747.

51. Shu XO, Moore DB, Cai Q, Cheng J, Wen W, Pierce L, Cai $\mathrm{H}$, Gao YT, Zheng W. Association of cyclin D1 genotype with breast cancer risk and survival. Cancer epidemiology, biomarkers \& prevention: a publication of the American Association for Cancer Research, cosponsored by the American Society of Preventive Oncology. 2005; 14:91-97.

52. Madrid-Paredes A, Canadas-Garre M, Sanchez-Pozo A, Segura-Perez AM, Chamorro-Santos C, Vergara-Alcaide E, Castillo-Portellano L, Calleja-Hernandez MA. ABCB1 C3435T gene polymorphism as a potential biomarker of clinical outcomes in HER2-positive breast cancer patients. Pharmacological research. 2016; 108:111-118.

53. Murali A, Varghese BT, Kumar RR, Kannan S. Combination of genetic variants in cyclin D1 and retinoblastoma genes predict clinical outcome in oral cancer patients. Tumor Biology. 2015; 37:3609-3617.

54. Okamoto K, Makino S, Yoshikawa Y, Takaki A, Nagatsuka Y, Ota M, Tamiya G, Kimura A, Bahram S, Inoko $\mathrm{H}$. Identification of I kappa BL as the second major histocompatibility complex-linked susceptibility locus for rheumatoid arthritis. American journal of human genetics. 2003; 72:303-312.

55. Modrek B, Resch A, Grasso C, Lee C. Genome-wide detection of alternative splicing in expressed sequences of human genes. Nucleic acids research. 2001; 29:2850-2859.

56. Wang M, Yuan L, Wu D, Zhang Z, Yin C, Fu G, Wei Q. A novel XPF $-357 \mathrm{~A}>\mathrm{C}$ polymorphism predicts risk and recurrence of bladder cancer. Oncogene. 2010; 29:1920-1928.

57. Lewis CM. Genetic association studies: design, analysis and interpretation. Brief Bioinform. 2002; 3:146-153. 